\title{
Detecting Deceptive Movement in 1 vs. 1 Based on Global Body Displacement of a Rugby Player
}

\author{
Sébastien Brault ${ }^{1,2}$, Benoit Bideau ${ }^{1}$, Richard Kulpa ${ }^{1}$ and Cathy Craig ${ }^{2}$ \\ ${ }^{I}$ M2S Laboratory, UFR APS, University of Rennes 2 - ENS Cachan, Avenue Charles Tillon, 35044 Rennes, France \\ ${ }^{2}$ School of Psychology, Queen's University of Belfast, Northern Ireland
}

\begin{abstract}
A key to success in many sports stems from the ability to anticipate what a player is going to do next. In sporting duels such as a 1 vs. 1 in rugby, the attacker can try and beat the defender by using deceptive movement. Those strategies involve an evolution of the centre of mass (COM) in the medio-lateral plane, from a minimal state to maximal displacement just before the final reorientation. The aim of this work is to consider this displacement as a motion-gap, as outlined in Tau theory, as a potential variable that may specify deceptive movement and as a means of comparing anticipatory performance between mid-level players and novices in rugby. Using a virtual reality set-up, 8 mid-level rugby players (ML) and 8 novices (NOV) observed deceptive (DM) and non-deceptive movements (NDM). The global framework used an occlusion time paradigm with four occlusion times. Participants had to judge the final direction of the attacker after the different cuts-off. For each movement and at each occlusion time, we coupled the ability to predict the good final direction with the value of the COM displacement in the medio-lateral (COM M/L) plane or with the Tau of this parameter (Tau COM). Firstly, results show that the Tau COM is a more predictive optical variable than the simple COM M/L. Secondly, this optical variable Tau COM is used by both groups, and finally, with a specific methodology we showed that mid-level players have significantly better anticipatory ability than the novice group.
\end{abstract}

Index Terms - Anticipation, Deceptive movement, Perception, Rugby, Tau.

\section{INTRODUCTION}

Perceptual skill is fundamental to successful performance in sport $[1,2]$. Numerous investigators in cognitive psychology examined visual strategy in sports [3, 4]. Those studies showed, in different sports, such as tennis [5] ice-hockey [6], badminton [7], squash [8] or football [9] that expert performers are believed to possess the ability to perceive determinant visual information from an opponent player's movement and use that information to anticipate subsequent events [10]. In brief, expert perception requires that players tune into the correct prospective visual information that specifies a key future event. In the past a variety of

Manuscript Received on 20 July 2008.

E-Mail: sebbrault@wanadoo.fr methodologies have been used to examine expertise at a perceptual level.

In one of those approaches researchers analysed the visual search pattern of a performer with eye movement registration techniques. This approach is assumed to identify important sources of information used by a subject. Nevertheless, the findings are contradictory [11]. For example, while authors showed that the position of the hips, kicking leg and trunk just before and during contact in penalty kick is presumed to be important information for expert goalkeepers $[12,13]$, others argue that the orientation of the non-kicking foot is key [14]. Those contradictions are mainly explained by the possibility to fixate on an object without extracting any specific information [15]. This has been referred to as the difference between "looking", which implies fixation on the fovea, and "seeing" which entails information processing [16]. Another explanation of this inconsistency stems from the possibility to fixate on a point in the field but extract information from the periphery [11]. Another approach has been to present a video display of an opponent's action and to integrate a temporal or a spatial occlusion in the movement. During temporal occlusion the movement is stopped at various times before or after a critical event and the subject has to predict the final outcome of the action [17]. Contrary to the eye tracking systems, results are less ambiguous. Thus, this methodology has been used in tennis [18, 19], in squash [20], or in karate Mori et al. [21]. In most of these studies, the movement's outcome is a ball trajectory that has to be analysed and interpreted. Therefore the coupling between trajectory data and decision-making processes was at the heart of these studies [22]. On that account, an original and interesting challenge is to analyse those perceptual skill but: i) without using an eye tracking system ii) looking at anticipation when no ball is involved and it is only the regulation of the movement of the opponent's body. This suggests that the explicit visual information of the situation is not as predictable as would be when a projectile is involved. This variability is due to the opportunity of the performer to modify his action online and thus create uncertainty about what he is going to do next. These situations, which are only regulated by body movements, are frequent in sports such as 1 vs. 1 duels in rugby when an attacker tries to beat the defender. The DM is one of those motor opportunities for the attacker to fool the defender. The aim is to mislead the defender by starting to move in one direction and then 
changing running direction at the last moment. The best parameter to highlight global body movement is the COM because it represents the outcome of the global body strategy. Thus, during DM, the COM displacement in the medio-lateral plane will reveal a reorientation phase. In others words the player has to control the closure of the gap between the initial state (direction of the movement before the DM) and the maximal state (maximal deviation in the wrong direction before reorientation).

Tau is a variable which refers to the evolution of a motion-gap [23]. The changing gap between two measurable states defines this motion-gap. Numerically, Tau is defined as the ratio of the current size, $x$, of the motion-gap and its current rate of closure, $\dot{x}$ such that $\tau(x)=x / \dot{x}$. Researchers have shown how it is possible to govern the timing of the initiation of a movement [24] or discriminate the time of arrival [25] using tau of the motion-gap between an object and its effector. Taken this into account it could therefore be conceptualised that the displacement of the tau of the COM gap could specify the time to reorientation of the body and be a variable that could be used to anticipate in which direction a player is going to run in.. In sport [26] have shown that tau theory can be used to understand the regulation of putting in expert golf players.

Consequently, in sporting scenarios, tau of a motion-gap is a variable which can influences the decision-making process and action. In the case of DMs, it may be possible to use this variable to anticipate a reorientation peak in the global body displacement and interpret the current direction as a wrong direction.

This paper aims to present a methodology to analyse in the virtual environment the perceptual activity of the defender in a 1 vs. 1 situation in rugby. Further, we are interested in a variable which can be used to detect deceptive movements. The hypothesis that we support is that, making reference to tau theory, the COM displacement in the medio-lateral plane can be used by the defender as a temporal variable to anticipate the point of body reorientation and consequently deceptive movement. Furthermore, with this approach it may be possible to show greater anticipation in mid-level rugby players than in a novice population.

\section{METHODS}

\subsection{Animation process and the virtual environment}

Deceptive movements were recorded from real 1 vs. 1 rugby situations using the optoelectronic motion capture Vicon MX camera system (Oxford Metrics, Oxford, UK). The animation process consisted of adapting in real-time those real captured movements to a new bones hierarchy in a virtual environment. The animation module used was MKM (Manageable Kinematic Motion) [27, 28]. It allows the captured movement to be adapted so that a virtual humanoid produces realistic movements. It has already been used and validated in movement simulations in different sporting duels [29, 30]. The final virtual reality solution is managed by the 3D development software Virtools 4.0 and all the developed components are integrated by Virtools (3D rugby pitch, humanoid animation, management of the interface). Participants viewed the virtual rugby stadium through two small screens inside a three-dimensional head mounted display (HMD) unit (Cybermind Visette pro, Cybermind Interactive Nederland, Maastricht, The Netherlands, $45^{\circ}$ each eye, resolution $1280 * 1024)$. This HMD was coupled in real time using the Vicon motion capture system (5 markers on the HMD) to allow head-movements to be tracked in real time updating instantly the displayed viewpoint in the virtual world.

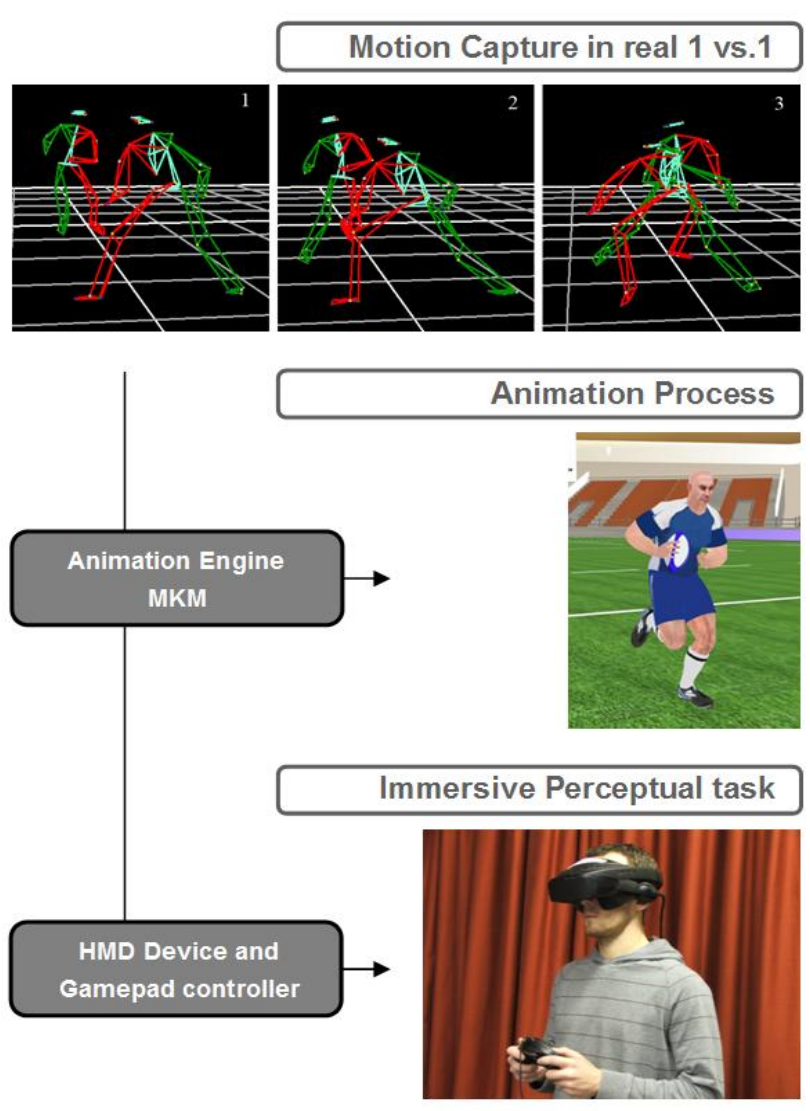

Fig. 1. Overview of the global framework: from motion capture to the immersive perceptual task

\subsection{Participants and the protocol in the virtual environment}

Eight ML rugby players (mean age 24.75 years; s.d. $=4.78$ ) who play in the French national league division 5, and eight NOV players (mean age 27.75 years; s.d. $=4.43$ ) took part in this experiment. The experiment employed a temporal occlusion paradigm. 14 movements were selected: five during which the attacker passed to the left of the defender by performing a deceptive movement (DM) to the right and five during which he passed to the right of the defender by performing a deceptive movement to the left. The other two attacks did not involve any DMs and were made up of two simple directional changes (NDM): one to the left and one to the right of the defender. Four occlusion times are carried out. The first one (T0) is made when the attacker's foot makes 
contact with the ground before changing direction (left foot for DM to the right and resp. right foot for DM to the left). The other occlusions were implemented at 100ms (T1), 200ms (T2) and 300ms (T3) after T0. As each participant was confronted with 14 movements*4 occlusions $* 4$ repetitions, they judged a total of 224 randomised movements. The subject made their predictions by pressing the appropriate button on a Microsoft sidewinder gamepad to indicate the final running direction of the attacker (right or left). A training period was carried out to familiarize subjects with the virtual environment and the task.

\section{Mid-level player $n^{\circ} 2$}
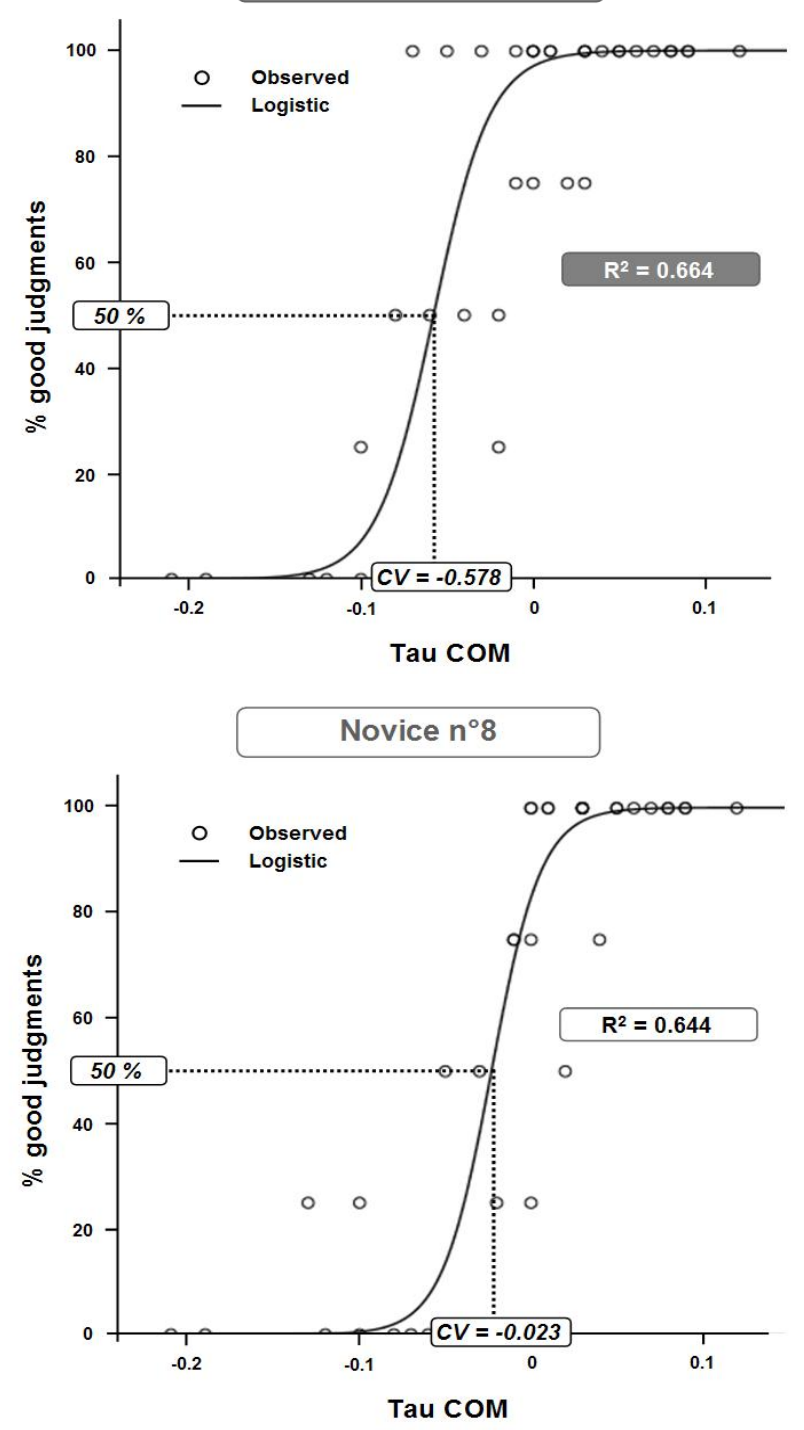

Fig. 2. Example of logistical regression between the Tau of the COM displacement in the $\mathrm{M} / \mathrm{L}$ plane and the $\%$ of good judgments. The Critical Value (CV) is obtained by projecting the $50 \%$ goal responses on the regression curve. The results for novice $\mathrm{n}^{\circ} 8$ and mid-level player $\mathrm{n}^{\circ} 2$ are presented here.

\subsection{Informational variable Tau and the analysis process}

The mean percentage of correct judgements was calculated per subject, per movement and for each occlusion time.
Those results were regressed with the COM position in the medio-lateral plane and with the tau of this measure using a logistic (S-shaped) function (' $a$ ' and ' $b$ ' are constant values and ' $u$ ' is the upper bound):

$$
f(x)=1 /\left((1 / u)+\left(a \times b^{x}\right)\right)
$$

Logistical regressions are described by a determination coefficient $\left(\mathrm{R}^{2}\right)$ which symbolizes the level of use of this variable to predict the final direction of the attacker. Then we calculate the critical value $(\mathrm{CV})$ of the information variable where $50 \%$ goal responses are predicted (Fig.2). As we explained before, the challenge of this work is to analyse perceptual skill when faced with the uncertainty of "human" movement. Given the inherent variability associated with this type of task comparisons yielding $\mathrm{R}^{2}$ values greater than 0.5 will be selected for between group comparisons. Consequently, the $\mathrm{CV}$ analysis will make sense only when the $\mathrm{R}^{2}$ are greater than 0.5 .

\subsection{Statistical analysis}

We compared the values for both the $\mathrm{R}^{2}$ and the $\mathrm{CV}$ s from the logistical regressions for the two groups using an independent groups Student T-test ( $\mathrm{p}<0.05)$.

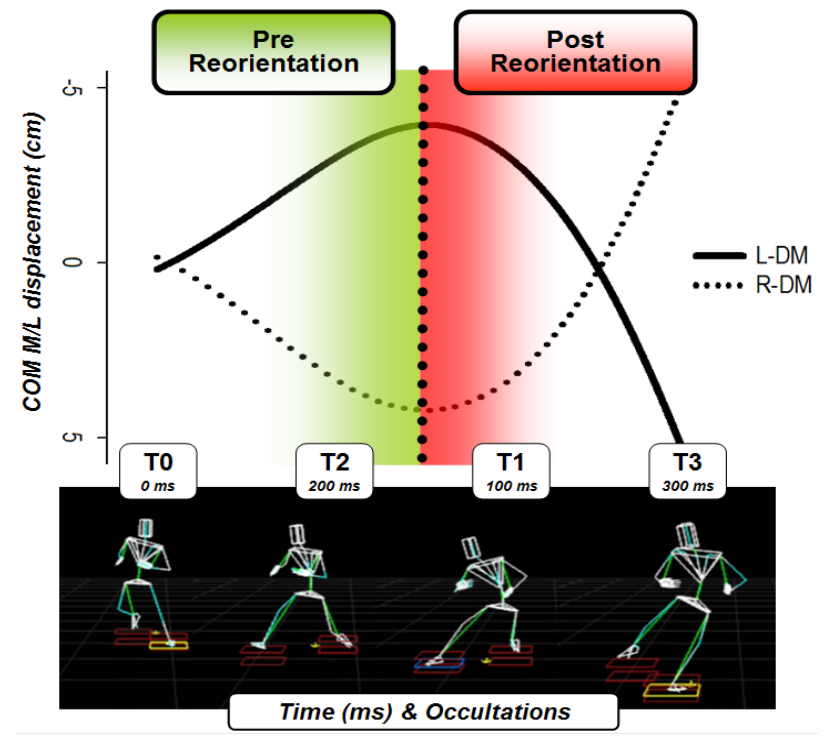

Fig. 3. Mean curve of the COM M/L displacement during L-DM \& R-DM (resp. DM on the Left \& DM on the Right).The vertical dot line symbolizes the reorientation peak.

\section{RESULTS}

\subsection{Medio-lateral displacement of the COM}

The COM displacement in the M/L plane shows logically a reorientation peak, which symbolises the transition between the wrong current direction and the real final direction (Fig.3). As we explained before, this parameter is probably not the main and the most accurate source of information, but in this global approach it seems to be the most relevant parameter to symbolise global body displacement. 


\subsection{Logistical regression analysis: $M L$ vs. NOV}

Table 1 shows the logistical regression results. On the one hand, they show that the simple COM displacement is presumably not a variable used by either of the groups (Mean $\mathrm{R}^{2}$ for NOVs $=0.09 \pm 0.02 \&$ for MLs $=0.023 \pm 0.005$ ). On the other hand, the

tau variable, which is calculated from the motion-gap created by this displacement, shows $\mathrm{R}^{2} \mathrm{~s}$ predominantly greater than 0.5 (Mean $R^{2}$ for NOVs $=0.55 \pm 0.03 \&$ for MLs $=0.50 \pm 0.04$ ). For this last variable, the comparison between both populations does not show any significant differences $(\mathrm{t}(1,14)=1,007 ; p=0,331)$ (Fig.5).

The CV's results can be projected on the mean curve of the tau evolution and thus, be used to estimate the time where the variable becomes more pertinent to detect DMs (Fig. 4) (\% good judgments $>50 \%)$. The time estimate results show a significant difference between ML and NOV $(\mathrm{t}(1,14)=2,585$; $p=0,022)$ (Fig.5) indicating that the ML group are picking up the relevant information earlier than the NOV group.

TABLE 1: DETERMINATION COEFFICIENT $\left(\mathrm{R}^{2}\right)$ AND CRITICAL VALUE (CV) OF THE LOGISTICAL REGRESSION OF THE M/L COM DISPLACEMENT AND OF THE TAU OF THIS COM DISPLACEMENT (TAU COM) FOR ML \& NOV

\begin{tabular}{|c|c|c|c|c|c|}
\hline \multirow[b]{2}{*}{ Groups } & \multicolumn{2}{|c|}{ COM (M/L) } & \multicolumn{3}{|c|}{ TAU COM (M/L) } \\
\hline & & $\mathrm{R}^{2}$ & $\mathrm{R}^{2}$ & $\begin{array}{l}\text { CV (50\% correct } \\
\text { judgments) }\end{array}$ & $\begin{array}{c}\text { Estimate } \\
\text { Times }\end{array}$ \\
\hline Novice & $M$ & 0,09 & 0,56 & $-2,68$ & 123,56 \\
\hline$(n=8)$ & SEM & 0,02 & 0,03 & 0,98 & 13,22 \\
\hline Mid-level & $M$ & 0,02 & 0,50 & $-6,80$ & 78,50 \\
\hline$(n=8)$ & SEM & 0,01 & 0,04 & 1,55 & 11,37 \\
\hline
\end{tabular}

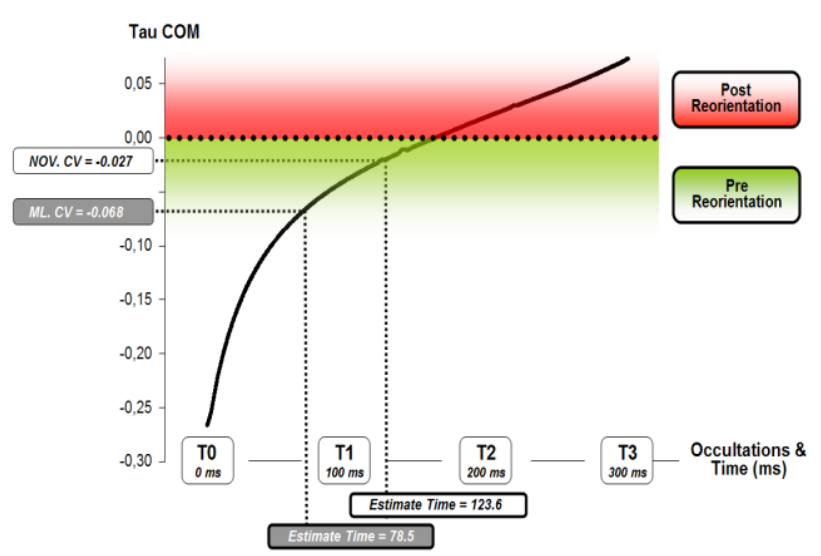

Fig. 4. Evolution of the Tau of the COM M/L displacement from occlusion T0 to T3. Estimate times of the critical value (NOV.CV \& ML.CV) for both groups are estimated. The horizontal dotted line (when Tau COM =0) symbolizes the reorientation peak.

\section{DISCUSSION}

This study does not aim to underline accurately the real sources of information used by a rugby defender, but instead it shows how, with a specific methodological approach, the global body displacements of an attacker can be used as a variable to predict $\mathrm{DM}$.

Findings showed that the simple COM displacement in the $\mathrm{M} / \mathrm{L}$ plane was not a variable used by both populations to infer future running direction from the current direction of the global body of the attacker (mean $\mathrm{R}^{2}$ for both groups = $0.058 \pm 0.0132$ ). However, making reference to tau theory, the analysis of this displacement as a spatio-temporal measure of a motion-gap, showed much more interesting results. Indeed, the Tau of the COM displacement in the $\mathrm{M} / \mathrm{L}$ plane yielded significantly greater determination coefficients (mean $\mathrm{R}^{2}$ for both groups $=0.529 \pm 0.0258$ ). Although this relationship only explains $53 \%$ of the variance in the data, it does show how a global parameter such as COM displacement can be used by most of the subjects (ML \& NOV) to make reliable anticipatory judgements from "human" movement. This variable seems to inform about the degree of credibility of the current direction of the attacker. That being said, the sensitivity of both populations to this variable was found to be significantly different. The analysis of the $\mathrm{CV}$ allowed us to highlight the mean threshold times when both groups modified their judgements as a function of this variable (50\% good judgements). These results underline significantly slower time estimates for the NOV group. It means that the NOV group needs a larger value of this variable to interpret the current direction as a wrong direction (in other words they are less sensitive to this variable). This suggests a greater sensitivity to this variable and greater anticipation skill for the ML group.
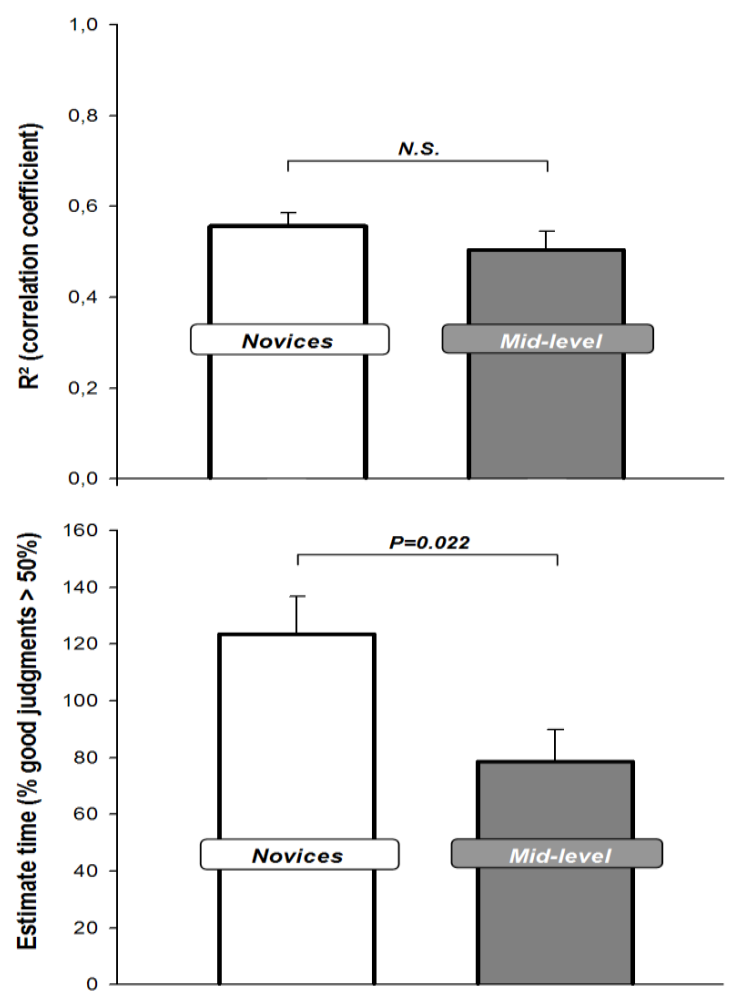

Fig. 5. Mean $\mathrm{R}^{2}$ and Estimate Times for both groups: ML and NOV. The error bars represent the standard errors. Statistical comparisons were performed using Student-T Tests $(\mathrm{p}<0.05)$. 
In conclusion, we have demonstrated how the global body displacement in the M/L plane, analysed using COM displacements, can be used by a defender (novice or mid-level player) to anticipate a reorientation peak and thus a DM. In addition, this work shows that this anticipatory skill is not only due to a specific visual search strategy but also to a greater sensitivity to the same spatio-temporal variable.

\section{ACKNOWLEDGEMENT}

This study was partially supported by the International Doctoral College of the European University of Brittany and the British Council Alliance Travel fund scheme.

\section{REFERENCES}

[1] B. Abernethy. Searching for the minimal essential information for skilled perception and action, Psychological Research, vol. 55, pp. 131-138, 1993.

[2] A. Williams, K. Davids and J. Williams. Visual perception and action in sport, Eds. London: E. \& F. N. Spon, 1999.

[3] B. Abernethy, A. Hanna and A. Plooy. The attentional demands of preferred and non-preferred gait patterns, Gait \& Posture, vol. 15, no. 3, pp. 256-265, 2002.

[4] A. Williams. Visual search behaviour in sport, Journal of Sports Sciences, vol. 20, no. 3, pp. 169-170, 2002.

[5] J. Shim, L. Carlton, and Y. Kwon. Perception of kinematic characteristics of tennis strokes for anticipating stroke type and direction, Research Quarterly for Exercise and Sport, vol. 77, no. 3, pp. 326-339, 2006.

[6] J. Salmela and P. Fiorito. Visual cues in ice hockey goaltending, Canadian journal of applied sport sciences, vol. 4, pp. 56-59, 1979.

[7] B. Abernethy. Dual-task methodology and motor skills research: Some applications and methodological constraints, Journal of Human Movement Studies, vol. 14, pp. 101-132, 1988.

[8] B. Abernethy, D. Gill, S. Parks and S. Packer. Expertise and the perception of kinematic and situational probability information, Perception, vol. 30, pp. 233-252, 2001.

[9] G. Savelsbergh, A. Williams, J. Van der kamp and P. Ward. Visual search, anticipation and expertise in soccer goalkeepers, Journal of Sports Sciences, vol. 20, no. 3, pp. 279-287, 2002.

[10] J. Shim, L. Carlton, J. Chow and W. Chae. The use of anticipatory visual cues by highly skilled tennis players, Journal of Motor Behaviour, vol. 37, no. 2, pp. 164-75, 2005.

[11] A. Williams. Perceptual skill in soccer : implications for talent identification and development, Journal of Sports Sciences, pp. 18, no. 9, pp. 737-750, 2000.

[12] D.A Tyldesley, R.J. Bootsma and G. Bomhop. Skill level and eye movements patterns in a sport-oriented reaction time task. In $\mathrm{H}$. Rieder, K. Bos, H. Mechling and K. Reische (Eds.), Motorik und bewegungsforchung, Schorndorf: Hofmann, 1982.

[13] A. Williams, and L. Burwitz. Advance cue utilization in soccer. In J. Clarys, T. Reilly and A. Stibbe (Eds.), Science and football II, London: E \& FN Spon, pp. 239-244, 1993.

[14] I.M. Franks and T. Hanvey. Cues for goalkeepers: high-tech methods used to measure penalty shot response, Soccer Journal, vol. 42, pp. 30-33, 1997

[15] J.M Papin, P. Metges and R. Amalberti. Use of NAC eye mark by radiologists. In A. Gale and F. Johnson (Eds.), Theoretical and applied aspects of eye movements research, Amsterdam: Elsevier Science, 1984.

[16] B. Abernethy. Visual search in sport and ergonomics: Its relationship to perceptive attention and performer expertise, Human Performance, vol. 4, pp. 205-235, 1988

[17] B. Abernethy and R. Russell. The relationship between expertise and visual search strategy in a racquet sport, Human movement science, vol. 3, pp. 283-319, 1987.

[18] C. Jones and T. Miles. Use of advance cues in predicting the flight of a lawn tennis ball, Journal of human movement studies, vol. 4, pp. 231-235, 1978.
[19] D. Scott, L. Scott, and B. Howe. Training Anticipation for Intermediate Tennis Players, Behavior Modification, vol. 22, no. 3, pp. 243, 1998

[20] B. Abernethy. Expertise, visual search and information pick-up in squash, Perception, vol. 19, pp. 63-77, 1990.

[21] S. Mori, Y. Ohtani and K. Imanaka. Reaction times and anticipatory skills of karate athletes, Human Movement Science, vol. 21, no. 2, pp. 213-230, 2002.

[22] C. Craig, E. Berton, G. Rao, L. Fernandez and R. Bootsma. Judging where a ball will go: the case of curved free kicks in football, Naturwissenschaften, vol. 93, no. 2, pp. 97-101, 2005.

[23] D.N. Lee. Guiding movement by coupling taus, Ecological Psychology, vol. 10, pp. 221-250, 1998.

[24] D.N. Lee and P.E Reddish. Plummeting gannets: a paradigm of ecological optics, Nature, vol. 293, pp. 293-294, 1981.

[25] D. Regan, and S. Hamstra. Dissociation of discrimination thresholds for time to contact and for rate of angular expansion, Vision Research, vol. 33, pp. 447-462, 1993.

[26] C.M. Craig, D. Delay, M.A. Grealy and D.N. Lee. Guiding the swing in golf putting, Nature, vol. 405, pp. 295-296, 2000.

[27] F. Multon, R. Kulpa and B. Bideau. MKM: a global framework for animating humans in virtual reality applications, Presence: Teleoperators and Virtual Environments, vol. 17, no. 1, pp. 17-28, 2008.

[28] R. Kulpa. Adaptation interactive et performante des mouvements d'humanoïdes synthétiques: aspects cinématique, cinétique et dynamique. Phd thesis. Rennes, France, 2005.

[29] B. Bideau, F. Multon, R. Kulpa, L. Fradet, B. Arnaldi and P. Delamarche. Using virtual reality to analyse links between handball thrower kinematics and goalkeeper's reactions, Neuroscience Letters, vol. 372, no.1-2, pp.119-122, 2004.

[30] B. Bideau, R. Kulpa, S. Menardais, L. Fradet, F. Multon, P. Delamarche and B. Arnaldi, Real handball goalkeeper vs. virtual handball thrower, Presence: Teleoperators and Virtual Environments, vol. 12, no. 4, pp. 411-421, 2003.

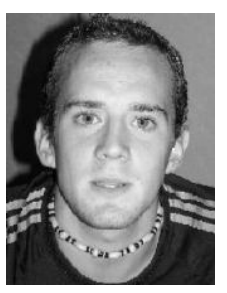

Sébastien Brault is currently a PhD Student studying Sports Science (biomechanics) at the M2S Laboratory (http://www.uhb.fr/labos/m2s) of the University of Rennes 2 (France) and is an international collaborator with the School of Psychology, Queen's University of Belfast. His PhD focuses on the application of virtual reality solutions to develop immersive sporting environments and thus, test and analyze perceptual skills. His research involves the use of biomechanical tools and perception/action analysis in order to understand the perceptual determinants of expertise in different sport's duel situations such as rugby.

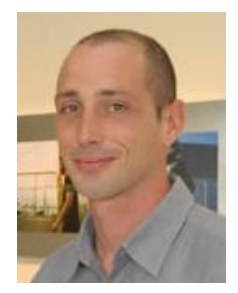

Benoit Bideau is a lecturer at the M2S laboratory, University of Rennes 2, France (http://www.uhb.fr/ labos $/ \mathrm{m} 2 \mathrm{~s})$. His research interests include biomechanics, perception in sport and virtual reality. Since 2000 he has been working on how to use virtual reality to study interactive behavior in different sports. One of his main goals is to provide realistic VR environments to enhance the level of presence and evaluate the influence the animation process (from motion capture to virtual human) has on improving the level of presence of immersed participants. His published studies show how handball goalkeepers react in the same way in a virtual environment as they do in a real environment. He has also shown how the MKM animation engine can be adapted to evaluate perceptual skills. He is currently developing virtual environments for different sports (rugby, handball, soccer) in order to evaluate anticipatory skill in top class athletes.

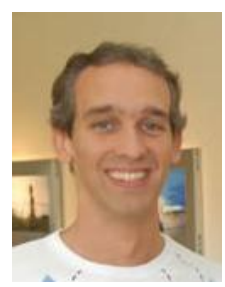

Richard Kulpa is a lecturer at the M2S laboratory, University of Rennes 2, France (http://www.uhb.fr/ $\underline{\text { labos } / \mathrm{m} 2 \mathrm{~s}}$ ). He is also an external collaborator of the Bunraku team of the IRISA-INRIA Rennes research institute (http://www.irisa.fr/bunraku). His research interests include biomechanics, realistic humanoids, animation and virtual reality. He worked since 1996 
as a research engineer in the SIAMES team (now Bunraku) where he worked on behavioral animations of characters in informed environments in collaboration with French multimedia companies. Since 2000, in parallel to these previous works, he has integrated the LPBEM laboratory where he worked on biomechanics and its use in IK methods in order to produce fast and realistic animations. At last, he received his PhD in 2005 for a work that combines biomechanics and iterative inverse kinematics and kinetics solver in order to have realistic animation of hundreds of characters in real-time. He received a price at Eurographics 2005 and FMX 2008 concerning this work. By the way, he co-created the MKM animation engine that incorporates all these works. He currently uses MKM in virtual reality applications in order to evaluate performance in sports.

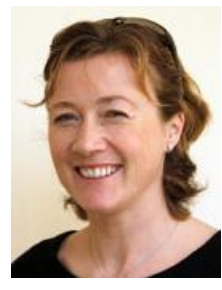

Cathy Craig is a Senior Lecturer in Visual Perception in the School of Psychology, Queen's University Belfast. Having obtained her Ph.D. at the University of Edinburgh, she went on to take up a post-doc at the Movement and Perception lab at the Sports Science Faculty at the University of Aix-Marseille 2. It was here that she first encountered virtual reality. The Movement and Perception lab was one of the first labs to use VR to study sports related skills. In a project funded by adidas she used VR to simulate curved free-kicks in soccer and investigate their effects on elite goal-keepers' anticipatory ability. Since taking up her current position at QUB, she has built an immersive interactive VR lab (https://www.qub.ac.uk/virtualreality) where she is continuing to use VR to investigate perceptual skills in different sports. 